\title{
Recognition and treatment of depression in older adults admitted to acute hospitals in England
}

\author{
Authors: Abhishek Shastri, ${ }^{\mathrm{A}}$ Lina Aimola, ${ }^{\mathrm{B}}$ Beatrice Tooke, ${ }^{\mathrm{C}}$ Alan Quirk, ${ }^{\mathrm{D}}$ Oliver Corrado, ${ }^{\mathrm{E}}$ Chloe Hood ${ }^{\mathrm{F}}$ \\ and Mike J Crawford ${ }^{\mathrm{G}}$
}

Levels of awareness and treatment of depression in older adults admitted to acute hospitals are unclear. This study aims to examine the proportion of older adults diagnosed with depression in acute hospitals, treatment, referral, and communication between secondary and primary healthcare services following discharge. Retrospective examination of records of 766 older adults admitted to 27 acute hospitals in England was carried out. Ninety-eight $(12.7 \%, 95 \%$ confidence interval $(\mathrm{CI})=10.6-15.3)$ records included a diagnosis of depression of which eight $(1.0 \%, 95 \% \mathrm{CI}=0.5-2.0)$ had a new diagnosis made during their hospital admission. All newly diagnosed and $76(84.4 \%, 95 \% \mathrm{CI}=75.5-90.5)$ of those with an existing diagnosis of depression were prescribed antidepressant medication. Six $(75.0 \%, 95 \% \mathrm{CI}=40.9-92.8)$ of those with a new diagnosis, and $21(23.3 \%, 95 \% \mathrm{CI}=15.8-33.0)$ with an existing diagnosis of depression were referred to liaison psychiatry. References to mental health were made in 50 (51.0\%, 95\% CI = 41.2-60.6) discharge letters sent to primary care. Very few older adults admitted to acute hospitals in this study were diagnosed with depression during their inpatient stay. Opportunities for improving the mental and physical health of such patients appear to be being missed.

KEYWORDS: Depression, older adults

\section{Introduction}

Depression is common among older adults in acute hospitals. Data collected from 14,632 patients in 47 studies indicates a prevalence of $29 \%$. Inpatients with depression have increased mortality during their admission ${ }^{2}$ and following their discharge from hospital. ${ }^{3,4}$ In addition to emotional distress and reduced quality of life that older adults with depression experience, inpatients with

Authors: ${ }^{\mathrm{A}}$ specialist registrar in general adult and old age psychiatry, Central and North West London NHS Foundation Trust, London, UK; B research fellow, Royal College of Psychiatrists, London, UK; ' project manager, Royal College of Psychiatrists, London, UK; D senior programme manager, Royal College of Psychiatrists,

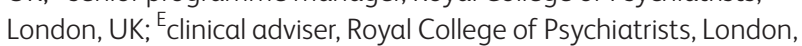
UK; F programme manager, Royal College of Psychiatrists, London, UK; ${ }^{G}$ director, Royal College of Psychiatrists, London, UK depression have longer lengths of stay in hospital and higher rates of readmission. ${ }^{4}$

It has been argued that an admission to hospital provides an important opportunity to review people's mental health and initiate treatment. NHS Scotland's Care of older people in hospital standards ${ }^{5}$ recommend that for patients with confirmed or possible depression, information about mental health is recorded in clinical records, a care plan is agreed and referral to psychiatric liaison or community mental health services is considered. The Royal College of Psychiatrists (RCPsych) recommends that mental health of all older adults admitted to acute hospitals should be routinely assessed and that all acute hospitals have an older adult psychiatric liaison team distinct from those provided for working age adults. ${ }^{1}$

Concerns have been raised that depression among older adults admitted to general hospitals is often unrecognised and untreated. ${ }^{6}$ Among a cross-sectional sample of 150 older adults admitted to a general hospital in Mississippi, Rapp and colleagues found that physicians were unaware of depression in over $90 \%$ of people suffering from this condition. In two Montreal hospitals, less than $50 \%$ of older medical inpatients with depression were recognised by physicians as being depressed. ${ }^{8}$ In a study from a tertiary academic medical centre in Switzerland, 15 out of 90 (16.7\%) older adults with depression on medical wards were detected by physicians, ${ }^{9}$ while in a study from Germany, only $21 \%$ of older adults admitted with depression received a diagnosis of depression on discharge. ${ }^{10}$ Koenig and colleagues ${ }^{11}$ examined treatment offered to 153 older adults with depression admitted to a general hospital in Durham, USA and found that about $60 \%$ of them did not receive antidepressant medication. Older adults with depression are also less likely to be referred for community-based psychological therapies, despite evidence they are more likely to attend sessions and benefit from these treatments. ${ }^{12,13}$

Over recent years there have been repeated calls to improve quality of mental health services for people who present to general medical services. ${ }^{14} \mathrm{NHS}$ England called for integrated treatment of physical and mental health conditions in the Five year forward view. ${ }^{15}$ While there are indications from studies conducted at single hospital sites in North America that recognition and treatment of depression among older adult inpatients is low, very little is known about levels of assessment and care received more widely. 8,16 In 2017 NHS England commissioned RCPsych to conduct an audit of recognition and treatment of depression among older adults in acute hospitals in England. We set out to assess and 
examine the proportion of older adults diagnosed with depression during their treatment in an acute hospital, how often referrals and treatments for depression were initiated, and quality of liaison between secondary care and primary care following their discharge.

\section{Methods}

This paper reports the results of a retrospective examination of clinical records of a cross section of older adults admitted to acute hospitals in England. We contacted audit leads at all NHS trusts in England that took part in the National Audit of Dementia in $2017^{17}$ and provided them with details of the study. We also publicised the study via the 72 members of Psychiatric Liaison Accreditation Network.

\section{Data collection}

Staff at each hospital that agreed to take part in the study were asked to audit the discharge summary and medical records of a consecutive sample of 30 patients aged 65 and over who had an unplanned admission to an acute hospital and were discharged after 1 April 2017. To be eligible for inclusion, patients needed to have been admitted for at least one night. We excluded patients if they had a coexisting diagnosis of dementia or slowly resolving delirium or if they died during their admission. Sites were asked to complete a copy of a survey tool for each patient.

A draft version of the survey tool was developed to address the aims of the study and minor changes were made following piloting at three hospitals. A copy of the survey tool is available from the corresponding author. Pseudonymised data were submitted using a bespoke online data portal. Data were then extracted and analysed using Predictive Analytics Software (PASW) Statistics 20/21. Data cleaning was carried out by identifying cases that did not meet sampling criteria, and checking for duplicates, unexpected values and outliers. Any suspected errors were emailed back to sites for checking against source data. We calculated proportions of those recognised and treated for depression and then estimated precision of these proportions by calculating $95 \%$ confidence limits. Online forms used to collect information were password protected. Each site was provided with a username and password, also held by the survey team at the RCPsych. Sites were asked to allocate codes to each eligible patient, which were used during data collection. It was stipulated that these should be independent codes that allowed sites to identify patients for the purposes of data cleaning and should not identify patients other than to those involved in data collection. The key to the patient codes was held by the site and was not known to the study team. We also sent a short questionnaire to each participating site concerning the organisation of services and whether the hospital had a specialist liaison service for older people.

\section{Outcome measures}

For each participant we asked trusts to return data on demographic details (age, gender, and ethnicity), type of ward where patients were predominantly treated on, length of stay in hospital, whether a history of depression was recorded at the time of admission and whether a new diagnosis of depression was made during treatment in hospital. Information was also extracted on whether there was a note of symptoms of depression in patient notes (regardless of whether they had a formal diagnosis of depression or not). Specific guidance was provided to consider symptoms such as persistent sadness or low mood, poor concentration or indecisiveness, agitation or slowing of movements, loss of interests or pleasure, low self-confidence, suicidal thoughts or acts or guilt. As biological symptoms of depression, such as fatigue or low energy, poor or increased appetite or disturbed sleep, also occur in a number of conditions affecting physical health, these were not included. Data about treatment of depression on admission were also collected, including prescription of antidepressant medication. Staff were also asked to record whether the patient was referred to mental health services during their admission to hospital and whether there was any reference to depression in the discharge summary sent to patients' general practitioner (GP) at the time of their discharge. Selected clinical practice standards derived from National Institute for Health and Care Excellence (NICE) guidelines CG90 ${ }^{18}$ and CG91, ${ }^{19}$ Quality Standards for Liaison Psychiatry Services ${ }^{20}$ and RCPsych guidelines for development of older adult liaison services ${ }^{1}$ were as follows.

$>$ Every older adult admitted to general hospital as an emergency should be asked about symptoms related to mental illness such as depression in order to recognise the condition.

> An appropriate antidepressant medication and/or psychological treatment should be considered for all depressed older people in acute hospitals.

> There should be a specialist mental health service for older adults in acute hospitals.

\section{Ethics}

Prior to the start of data collection, we followed national guidelines on whether projects require ethical review (www.hradecisiontools.org.uk/research/question4.html). As an audit, the approach we planned for collecting and analysing pseudonymised data did not need independent ethical review. Furthermore, each participating site was sent a report comparing the data they submitted with the national results, to assist them in improving care at these sites.

\section{Results}

Twenty-nine sites registered to take part in the audit. Two sites did not sample according to our predefined criteria, so data from these sites were excluded. Analysis was carried out on 766 eligible cases from 27 sites. Each site submitted data on a median of 30 patients (range $=14$ to 30 patients).

The mean age of the sample was 79 years (range $=65$ to 99 years). Approximately half the sample were female ( $n=414$, $54.0 \%)$ and most were white British ( $n=643,83.9 \%)$. Most $(76.8 \%)$ were admitted to hospital for between 1 and 10 days. Participants were admitted to a wide range of different wards, the most frequent being general medical ( $n=155,20.2 \%)$, care of the elderly ( $n=141,18.4 \%)$, general surgical $(n=111,14.5 \%)$ and cardiac care wards $(n=73,9.5 \%)$.

Most patients ( $n=631,82.3 \%, 95 \%$ confidence interval $(C I)=79.5-84.9 \%)$ had no record of the presence or absence of depression or depressive symptoms in their notes. Ninetyeight $(12.7 \%, 95 \% \mathrm{CI}=10.6-15.3 \%)$ of the 766 patients had a diagnosis of depression made in their clinical records when they were either admitted or discharged from hospital. Of these, 
Table 1. Diagnosis, treatment, referral of depression based on $\mathbf{7 6 6}$ patient records and communication with primary health care services

\begin{tabular}{llll} 
Treatment and referral & $\begin{array}{l}\text { New diagnosis of } \\
\text { depression, } n=8\end{array}$ & $\begin{array}{l}\text { Existing diagnosis of } \\
\text { depression, } n=90\end{array}$ & $\begin{array}{l}\text { No diagnosis of } \\
\text { depression, } n=668\end{array}$ \\
Prescribed antidepressant, $n(\%)[95 \% \mathrm{CI}]$ & $8(100.0)[67.5-100.0]$ & $76(84.4)[75.5-90.5]$ & $47(7.0)[5.3-9.2]$ \\
New prescription of antidepressant, $\mathrm{n}(\%)[95 \% \mathrm{CI}]$ & $7(87.5)[52.9-97.7]$ & $7(7.7)[3.8-15.1]$ & $4(0.6)[0.2-1.5]$ \\
Referral to liaison service, $\mathrm{n}(\%)[95 \% \mathrm{CI}]$ & $6(75.0)[40.9-92.8]$ & $21(23.3)[15.8-33.0]$ & $8(1.2)[0.6-2.3]$ \\
Reference to depression in discharge letter, $\mathrm{n}(\%)[95 \% \mathrm{CI}]$ & $8(100.0)[67.5-100.0]$ & $42(46.6)[36.7-56.9]$ & - \\
\hline $95 \% \mathrm{CI}=95 \%$ confidence interval. & & &
\end{tabular}

$90(11.7 \%, 95 \%$ CI $=9.6-14.2 \%)$ had a diagnosis of depression recorded at the time of admission and eight $(1.0 \%, 95 \% \mathrm{CI}=$ $0.5-2.0 \%$ ) had a diagnosis of depression made during admission. Of the 27 hospital sites, a new diagnosis of depression was made in seven sites, with one site making two new diagnosis of depression. No new diagnosis was made at the other 20 hospitals. In those with either an existing or new diagnosis of depression, 50 patients $(51.0 \%, 95 \% \mathrm{CI}=41.2-60.6 \%)$ had this recorded in their discharge notes/letters.

All eight patients who received a new diagnosis of depression during their admission were prescribed antidepressant medication. Of those with an existing diagnosis of depression, $76(84.4 \%$, $95 \% \mathrm{CI}=75.5-90.5 \%$ ) were prescribed antidepressants (Table 1 ). No patients with a new or existing diagnosis of depression were referred to psychological services.

Thirty-five of the 766 patients were referred to psychiatric liaison services including six (75.0\%) of the eight patients newly diagnosed with depression, and 21 (23.3\%) of the 90 patients with an existing diagnosis. These referrals all took place at 15 (55.5\%) of the 27 hospital sites. Eighteen of the 19 sites returned a completed organisational checklist, out of which 18 reported having a specific older adult liaison service.

In addition to those who had a diagnosis of depression documented in their notes, 37 patients $(4.8 \%, 95 \%$ $\mathrm{CI}=3.5-6.5 \%)$ had documented evidence of a discussion about depressive symptoms without getting a diagnosis. Of these, $15(40.5 \%, 95 \% \mathrm{CI}=26.3-56.5 \%)$ were either prescribed medication or referred to specialist mental health services during admission and five $(13.5 \%, 95 \% \mathrm{CI}=5.9-27.9 \%)$ were referred to psychiatric liaison services (Table 1).

\section{Discussion}

Results of this survey show that levels of depression recorded in clinical notes of people aged over 65 admitted to acute hospitals are lower than what would be expected given the high prevalence of this condition. With $13 \%$ of the study sample having a diagnosis of depression in their records, compared with other UK studies of prevalence such as $23 \%$ by Burn et al, ${ }^{21} 17.7 \%$ by Cullum et al, ${ }^{16}$ $8-32 \%$ by Goldberg et al, ${ }^{22} 35 \%$ by Jackson and Baldwin ${ }^{23}$ and $9.2 \%$ by Bowler et al, ${ }^{24}$ results of this study suggest that patients with depression in acute hospitals are under-recognised. In most instances where depression was recorded during hospital stay, this was because people already had a diagnosis of depression prior to admission. Instances of people having this diagnosis made while in hospital were very rare. In 20 of the 27 hospitals no new diagnosis of depression was made during hospital stay.
While most people with a diagnosis of depression were prescribed antidepressant medication, no patient with a diagnosis of depression was referred for psychological treatments. This is noteworthy as NICE guidelines clearly suggest using psychological therapies in the first instance for mild-to-moderate depression, as well as being part of management for moderateto-severe depression. Older adults benefit from being referred to psychological services such as Improving Access to Psychological Therapies (IAPT) services, but the proportion of older adults referred to such services are lower than younger adults. ${ }^{12,25,26}$

We also found that a diagnosis of depression was not systematically recorded in discharge notes, suggesting that depression is not being well communicated by hospital staff to GPs and primary care teams when patients leave hospital, thereby impacting their future care.

Many patients in this survey had some discussion of depression in their records. Of these, some had no diagnosis recorded but were prescribed antidepressant medication. It is therefore likely that these patients could have had a diagnosis of depression, but not properly recorded in their notes. Furthermore, some of the patients who had a discussion of depression in their notes did not receive medication or specialist referral.

\section{Study strengths and limitations}

This is the first time that data on recognition and treatment of depression have been gathered from across England. Our sample of over 700 patients allowed us to calculate proportions of patients whose depression was recognised and treated with a high degree of precision. By collecting data from 27 hospitals spread throughout England we have been able to generate data that are more generalisable than those conducted in previous, singlecentre studies. Out of the 29 sites that volunteered to take part in the study, eligible data were submitted by 27 sites.

However, the study has a number of limitations which need to be considered when interpreting the findings. Firstly, hospital sites were self-selected, and it is possible that practice in these hospitals is better or worse than that in hospitals that did not take part. As a case note audit, we were only able to report on information that was documented by staff. While it is possible that some discussions of patients' mental health were not documented, feedback from staff in acute hospitals suggests that people would not receive a new diagnosis of depression or be referred to specialist services without this being documented in their clinical records.

The questions regarding medication were designed to identify patients prescribed medication for depression only. However, 
sites reported it was sometimes difficult to determine this as the indication was not specified in notes. It is possible that medication prescribed for other reasons, for example chronic pain, was mistakenly included despite guidance to include medications prescribed for depression only. This would have led to us overestimating the proportion of people with depression who were prescribed antidepressant medication.

\section{Implications for patients, services and research}

These results add to the evidence that depression is often not recognised or treated among older adults admitted to acute hospitals and that national guidelines regarding treatment of inpatients with depression are not being followed. ${ }^{19}$ In recent years efforts have been made to ensure that mental health conditions are considered alongside people's physical health as an essential component of efforts to provide 'whole patient care.14,27,28

In order to reduce distress and disability that older adults with depression experience, staff in acute hospitals must be trained in recognition and management of elderly patients who may have depression. Hospitals must also have clear referral pathways in place to ensure that people with depression receive input from psychiatric liaison services for older people. This should facilitate specialist assessment, diagnosis, future care for emergency, urgent and routine referrals in accordance with guidance based on urgent and emergency liaison mental health services for adults and older adults issued by NHS England, NICE and the National Collaborating Centre for Mental Health. ${ }^{29}$ Liaison psychiatry teams also have a role to play in raising awareness of depression among older adults admitted to acute hospital and encouraging colleagues to assess the mental health of older adults. However, the results of this study suggest that the presence of an older adult liaison service in a hospital is not in itself sufficient to ensure that depression among older adults is properly assessed and treated. In addition to prescription of antidepressant medication, staff working in general hospitals should be aware of how people with depression can be referred for psychological treatments. In England, access to psychological therapies can be made directly through to IAPT ${ }^{26}$ or via the person's GP. However, in order for this to happen concerns about patient's mental health need to be included in communications between the hospital and the patients' primary care team.

A diagnosis of depression should be included in correspondence to primary care teams on discharge to improve continuity of care. Steps must be taken to ensure information about patients with existing mental health problems, including medication, can be shared seamlessly between primary care, acute hospitals and mental health services to assure quality and continuity of care upon arrival at the accident and emergency department or upon admission to, and discharge from, hospital. Clinical records should be updated by acute hospital staff following any relevant assessments, interventions and treatment for depression in hospital. These should be well-documented and included in discharge summaries to primary care services.

Further research is required in this area, especially around barriers to recognition of depression. Some known barriers include stigma, physician knowledge and limited time. ${ }^{30}$ One of the important factors in treatment of older adults is the concept of 'therapeutic nihilism' - the erroneous belief among some clinicians and patients that nothing can be done. ${ }^{31}$
Screening tools may be useful in recognising depression, but this would require training of clinicians in using validated tools. NICE guidelines recommend the use of screening for people at high risk of depression in adults with chronic physical health problems. These two questions have also been validated for older adults in acute hospital settings. ${ }^{32}$ Furthermore, the Edinburgh Depression Scale has also been validated for use in this population ${ }^{33}$ with the added advantage of including a question on self-harm (older people with depression are at a high risk of suicide). A systematic review by Dennis et $a{ }^{34}$ found that the Geriatric Depression Scale is the best instrument to screen for depression in older adults on acute hospital wards.

We conclude that recognition and treatment of depression in older adults on acute hospital wards in England falls below expected standards. Communication between primary and secondary health services is also poor. Health services, patients and researchers need to collaborate and enhance awareness of depression among older adults admitted to general hospitals if treatment of this condition is to improve.

\section{Funding}

This audit was funded by NHS England and conducted by staff at the College Centre for Quality Improvement at the Royal College of Psychiatrists. The views expressed in this article are those of the authors and not necessarily those of NHS England or the Department of Health.

\section{References}

1 Royal College of Psychiatrists. Who cares wins. Improving the outcome for older people admitted to the general hospital: Guidelines for the development of Liaison Mental Health Services for older people. London: RCPsych, 2005.

2 Cullum S, Metcalfe C, Todd C, Brayne C. Does depression predict adverse outcomes for older medical inpatients? A prospective cohort study of individuals screened for a trial. Age Ageing 2008;37:690-5.

3 Arfken CL, Lichtenberg PA, Tancer ME. Cognitive impairment and depression predict mortality in medically ill older adults. J Gerontol A Biol Sci Med Sci 1999;54:M152-6.

4 Covinsky KE, Kahana E, Chin MH et al. Depressive symptoms and 3 -year mortality in older hospitalized medical patients. Ann Intern Med 1999:130:563-9.

5 Health Improvement Scotland. Care of older people in hospital standards. Edinburgh: HIS, 2015. www.healthcareimprovementscotland.org/our work/person-centred care/resources/ opah_standards.aspx [Accessed 25 June 2018].

6 Cole MG, Bellavance F. Depression in elderly medical inpatients: a meta-analysis of outcomes. CMAJ 1997;157:1055-60.

7 Rapp SR, Parisi SA, Walsh DA. Psychological dysfunction and physical health among elderly medical inpatients. J Consult Clin Psychol 1988;56:851-5.

8 Cepoiu M, McCusker J, Cole MG, Sewitch M, Ciampi A. Recognition of depression in older medical inpatients. J Gen Intern Med 2007;22:559-64.

9 Pouget R, Yersin B, Wietlisbach V, Burnand B, Bula C]. Depressed mood in a cohort of elderly medical inpatients: Prevalence, clinical correlates and recognition rate. Aging (Milano) 2000;12:301-7.

10 Topitz A, Benda N, Saumer G et al. [Prevalence and recognition of depression among inpatients of non-psychiatric hospital departments]. Neuropsychiatr 2015;29:63-70.

11 Koenig HG, George LK, Meador KG. Use of antidepressants by nonpsychiatrists in the treatment of medically ill hospitalized depressed elderly patients. Am J Psychiatry 1997:154:1369-75. 
12 Prina AM, Marioni RE, Hammond GC et al. Improving access to psychological therapies and older people: Findings from the Eastern Region. Behav Res Ther 2014;56:75-81.

13 Pettit S, Qureshi A, Lee W et al. Variation in referral and access to new psychological therapy services by age: an empirical quantitative study. Br J Gen Pract 2017;67:e453-9.

14 Royal College of Psychiatrists. Whole-person care: from rhetoric to reality (Achieving parity between mental and physical health). London: RPsych, 2013.

15 NHS England. Five year forward view. London: NHS, 2014. www. england.nhs.uk/wp-content/uploads/2014/10/5yfv-web.pdf [Accessed 23 June 2018].

16 Cullum S, Tucker S, Todd C, Brayne C. Screening for depression in older medical inpatients. Int ] Geriatr Psychiatry 2006;21:469-76.

17 Royal College of Psychiatrists. National Audit of Dementia. London: RPsych, 2017. www.rcpsych.ac.uk/quality/nationalclinicalaudits/ dementia/nationalauditofdementia.aspx [Accessed 20 May 2018].

18 National Institute for Health and Care Excellence. Depression in adults: recognition and management. Clinical guideline [CG90]. London: NICE, 2009. www.nice.org.uk/guidance/cg90 [Accessed 21 September 2018].

19 National Institute for Health and Care Excellence. Depression in adults with a chronic physical health problem: recognition and management. Clinical guideline [CG91]. London: NICE, 2009. www. nice.org.uk/guidance/cg91 [Accessed 21 September 2018].

20 Psychiatric Liaison Accreditation Network. Quality standards for liaison psychiatry services. London: PLSA, 2017. www.rcpsych.ac.uk/ workinpsychiatry/qualityimprovement/ccqiprojects/liaisonpsychiatry/plan/planstandards.aspx [Accessed 21 September 2018].

21 Burn WK, Davies KN, Mckenzie FR, Brothwell JA, Wattis JP. The prevalence of psychiatric illness in acute geriatric admissions. Int J Geriatr Psychiatry 1993:8:171-4.

22 Goldberg SE, Whittamore KH, Harwood RH et al. The prevalence of mental health problems among older adults admitted as an emergency to a general hospital. Age Ageing 2012;41:80-6.

23 Jackson R, Baldwin B. Detecting depression in elderly medically ill patients: the use of Geriatric Depression Scale compared with medical and nursing observations. Age Ageing 1993;22:349-53.

24 Bowler C, Boyle A, Branford M et al. Detection of psychiatric disorders in elderly medical inpatients. Age Ageing 1994:23:307-11.
25 Chaplin R, Farquharson L, Clapp M, Crawford M. Comparison of access, outcomes and experiences of older adults and working age adults in psychological therapy. Int J Geriatr Psychiatry 2015:30:178-84.

26 National Collaborating Centre for Mental Health. The improving access to psychological therapies manual. London: NCCMH, 2018. www.england.nhs.uk/wp-content/uploads/2018/06/the-iaptmanual.pdf [Accessed 26 June 2018].

27 Rayner L, Matcham F, Hutton J et al. Embedding integrated mental health assessment and management in general hospital settings: feasibility, acceptability and the prevalence of common mental disorder. Gen Hosp Psychiatry 2014;36:318-24.

28 World Health Organization, Regional Office for the Eastern Mediterranean. Scaling up mental health care: a framework for action. WHO, 2015. www.who.int/iris/handle/10665/250492 [Accessed 20 June 2018].

29 National Collaborating Centre for Mental Health. Liaison Mental Health Services for Adults and Older Adults. London: NCCMH, 2016. www.england.nhs.uk/wp-content/uploads/2016/11/Imhsguidance.pdf [Accessed 18 June 2018].

30 Goldman LS, Nielsen NH, Champion HC. Awareness, diagnosis, and treatment of depression. J Gen Intern Med 1999;14:569-80.

31 Burroughs H, Lovell K, Morley M et al. 'Justifiable depression': how primary care professionals and patients view late-life depression? A qualitative study. Fam Pract 2006;23:369-77.

32 Esiwe C, Baillon S, Rajkonwar A et al. Screening for depression in older adults on an acute medical ward: the validity of NICE guidance in using two questions. Age Ageing 2015;44:771-5.

33 Esiwe C, Baillon S, Rajkonwar A et al. Screening for depression in older people on acute medical wards: the validity of the Edinburgh Depression Scale. Age Ageing 2016:45:554-8.

34 Dennis M, Kadri A, Coffey J. Depression in older people in the general hospital: a systematic review of screening instruments. Age Ageing 2012;41:148-54.

Address for correspondence: Dr Abhishek Shastri, Westminster Older Adults Community Mental Health Team, 190 Vauxhall Bridge Road, London SW1V 1DX.

Email: abhishek.shastri@nhs.net 